As compared to isolated males, the growths of castrated males kept in mixed pens were faster. It may therefore be assumed that the domination behaviour of males resulted in an increased feed intake in mixed pens to the expense of the females. Mixed rearing did not affect the mean performance (feed intake, growth, feed efficiency), but slightly improved their homogeneity. The body composition and commercial grading were most affected in males kept in mixed pens. It may therefore be concluded that from a practical point of view, sex separation leads to a better control of the carcass quality of males. Moreover from an experimental point of view, the results of the comparison between diets offered according to a restricted feeding system adapted to sex can be transposed in practice to mixed group rearing of females and castrated males.

\title{
Results of an inquiry about the quality of pig feeds in Brittany
}

\author{
L. HOUEIX *, P. LATIMIER **, J.-G. POILPRE*** \\ et J. SAULNIER ***** \\ * E.D.E. du Morbihan, B.P. 77, F 56000 Vannes \\ ** E.D.E. des Côtes-du-Nord, B.P. 54, F 22190 Plerin \\ **** E.D.E. dlllle-et-Vilaine, 22, avenue Janvier, F 35000 Rennes \\ ***** I.T.P., B.P. 3, La Motte-au-Vicomte, F 35650 Le Rheu
}

With the aim of determining the values of pig feeds used in Brittany, 134 commercial or farm produced feeds were sampled between the 12th and the 20th of November 1979 and subjected to the following analyses : moisture, total crude protein, fat, crude fibre, mineral matter, $\mathrm{Ca}$ and $\mathrm{P}$ [growing pig (PC) $n=53$; in gestating and lactating sow diets (T) $n=47$; piglet diets from 35 to 60 days (PLT) $n=22$; protein supplement for sows $n=12$ ].

In the commercial feeds the average moisture levels were high (PC: $14.08 \mathrm{p} .100$ $\pm 0.93 ; \mathrm{T} 13.61 \pm 0.85$ ) and exceeded by $14 \mathrm{p} .100$ the legal maximum in $50 \mathrm{p} .100$ and 30 p. 100 of the cases, respectively. The crude protein contents (PC 17.18 p. $100 \pm 0.72$; T $15.85 \pm 0.87$; PLT $18.09 \pm 1.34$ ) as well as lysine contents (PC $0.84 \pm 0.05 ; \mathrm{T} 0.77$ \pm 0.065 ; PLT $0.99 \pm 0.07$ ) were satisfactory, except for the PLT feeds. The energy values (UF/100 kg) assessed ( $\mathrm{PC} 99 \pm 2.7 ; \mathrm{T} 93 \pm 3.8 ; \mathrm{PLT} 101.5 \pm 2.3$ ) were extremely variable and quite insufficient for the lactation feeds $(T)$; there were therefore large differences in the lysine/energy ratios and no relationship between the quality of the feeds (energy value) and their prices. Moreover, the supplements for breeding animals were characterized by the differences in the protein levels (from 27.3 to $41 \mathrm{p}$. 100) and the incorporation level (from 16 to 40 p. 100) in the feeds.

The farm produced feeds were comparable to the industrial feeds, but with lower and more variable crude protein levels (PC 16.19 $\pm 1.08 ; T$ 15.56 $\pm 1.36 ;$ PLT 18.08 \pm 2.45 ).

A better knowledge and utilization of raw materials as well as a more detailed labelling of industrial feeds should contribute to improve their use by the farmers. 